\title{
Healthcare and the Coming Informatics Revolution
}

\author{
J. Christopher Westland \\ Professor, Department of Information \& Decision Sciences, University of Illinois- \\ Chicago
}

\begin{abstract}
Healthcare is the largest industry in the world, and set to grow even larger as the world's population ages. Presently, the US leads the world in medical technology; it also spends twice as much per capita on healthcare as other developed nations, the result of inefficiencies in the system and the increasing demands of risk management as medicine grows technologically more complex. The total cost of the US system is \$2 trillion annually or around $16 \%$ of US GDP, and employs 14 million people, or about $10 \%$ of the US labor force.
\end{abstract}

Other nations - India, China, Brazil, Thailand - offer world-class centers of excellence to rival the US. It is likely that in doing so, other nations will increasingly invite many of the same problems currently facing US healthcare if they do not carefully manage the integration of informatics and technology into healthcare practice. Different regions of the world are witnessing rapidly changing economic, health and demographic landscapes, with most of the world (including China) facing a rapid decline in birthrate and aging of the population over the near future. These changes are apt to tax the healthcare resources of those nations unless significantly greater efficiencies can be wrung from existing facilities, equipment and doctors. Fortunately, automation and advances in information technology offer these efficiencies. Information technology is still at a very early stage of application to healthcare in comparison to its successes over the past two decades in manufacturing, finance and logistics.

This talk will describe the opportunities available for automation and information technology applications to building an integrated national healthcare platform addressing three tasks

1. Practice management; for example of (1) asset centers such as hospitals, clinics and offices; (2) activities such as diagnosis, procedures and prescriptions; or drivers of demand, such as the health system, population health;

2. Innovation management; for example in (1) asset centers such as labs, universities, clinical trials and pharmaceuticals firms; (2) activities such as discovery, creation, $\mathrm{R} \& \mathrm{D}$, and markets for medical innovations and drugs; or (3) drivers of supply, such as funding and innovators;

3. Risk Management; for example in (1) asset centers such as insurance, legal, evidence databases and best-practice databases; (2) activities such as statistical mining of bestpractices, diagnoses, and patient education, social networking and information search; and (3) drivers of law and regulation such as human rights, healthcare 'events' and public health. 
